\title{
Usability Evaluation of E-Motion: A Virtual Rehabilitation System Designed to Demonstrate, Instruct and Monitor a Therapeutic Exercise Programme
}

\author{
Diarmaid Fitzgerald, Dan Kelly, Tomas Ward, Charles Markham and Brian Caulfield
}

\begin{abstract}
The importance of systematic usability evaluation of virtual rehabilitation systems cannot be underestimated. We have developed a virtual rehabilitation system with the functionality to guide a user through a therapeutic exercise programme. Progression is determined by users' ability to replicate movements as demonstrated by an on-screen character. Visual and auditory corrective feedback is provided during exercise in order to improve the user's postural control and biomechanical alignment. The objective of this study was to evaluate the usability of our system and subsequently implement modifications aimed at improving fidelity and ease of use. The first stage of our evaluation involved conducting an expert walkthrough with six experts currently researching in areas related to the system design. Following system refinement and modification we conducted a user evaluation study with twelve novice users using VRUSE, a computerised questionnaire-based usability evaluation tool for assessment of virtual environments. Results have provided a systematic evaluation of the system, provided information for guidance on system alterations and will allow comparison of usability levels with similar virtual rehabilitation systems tested with the same protocol.
\end{abstract}

\section{INTRODUCTION}

$\mathrm{U}$ SABILITY evaluation of virtual rehabilitation systems (VRS) is essential to ensure systems meet both design specifications and user requirement criteria. Recently there has been much research on the design of these systems for physical rehabilitation and studies have begun to investigate the clinical outcomes in patient groups [1-5]. However there has been a dearth of published research into usability evaluation of these systems. This paper gives an account of a usability evaluation of our prototype virtual rehabilitation system which may aid others in undertaking similar endeavors.

In the field of physical medicine and rehabilitation, VSR are increasingly being implemented to motivate and

Manuscript received June 30, 2008. This work was supported in part by the Higher Education Authority of Ireland.

Diarmaid Fitzgerald, and Brian Caulfield are with the School of Physiotherapy and Performance Science, Health Sciences Centre, University College Dublin, Belfield, Dublin 4, Ireland (phone: 00353-17166500; fax: 00353-1-7166501; e-mail: diarmaid.m.fitzgerald@ucd.ie; b.caulfield@ucd.ie).

Dan Kelly and Charles Markham are with the Computer Science Department, National University of Ireland Maynooth, Maynooth, Co. Kildare, Ireland (e-mail: dankelly@.cs.nuim.ie; cmarkham@.cs.may.ie;).

Tomas Ward is with Department of Electronic Engineering, National University of Ireland Maynooth, Maynooth, Co. Kildare, Ireland (e-mail: tomas.ward@nuim.ie). monitor patients' exercise programmes [6]. Exercise therapy is essential for the restoration of normal movement patterns in a range of patient groups such as musculoskeletal injuries, neurological pathologies and elderly care. Common problems encountered when these therapeutic exercise programmes are prescribed to patients are inadequate exercise performance due to lack of knowledge of the correct biomechanical technique and low compliance rates due to many factors such as poor motivation [7].

The aim of E-Motion, the VRS under examination here, was to develop a tool to teach and monitor a patients' therapeutic exercise programme by tracking whole body movements and by harnessing the interactive benefits of virtual reality technology and software. The system utilises a custom built motion tracking suit interfaced with a computer game designed to provide audio and visual feedback during exercise as described previously [8]. Ten inertial motion tracking sensors (MTx Motion Tracker, Xsens Technologies, The Netherlands) are worn by the user to track 3D body movements (Fig 1a) and data is transmitted wirelessly to a laptop using bluetooth technology. Users standing on an exercise mat and attempt to replicate the movements of the on-screen virtual personal trainer (VPT) (Fig 2). A projector (Dell 3400MP Analog RGB $1600 \times 1200$ ) is used to display the on-screen virtual environment onto a rear projection screen (ST-Professional-DC, $230 \mathrm{~cm} \times 174 \mathrm{~cm} \times 1.6 \mathrm{~cm}$, Screen Tech, Hamburg, Germany) and speakers (Samsung SMS-7841 Magic Spekers) provide the audio projection.

Our prototype game for the system, E-Yoga, is based on yoga exercise's which has shown many health benefits [9] and is increasingly being utilised for therapeutic purposes. We chose a yoga sequence known as the sun salutation sequence for this version of the game which is comprised of twelve poses completed one after the other (Fig 3). To create a scoring system in the game we recorded the movements of a master yoga teacher while wearing the motion capture suit and this provided the "perfect movement profile" against which users are compared during game play. The accuracy level for progression is adjustable thus providing a variable difficulty level for the game. This facilitates progression of exercise programmes as patients improve their performance and the creation of adaptable patient goals during their programme. 
(a)

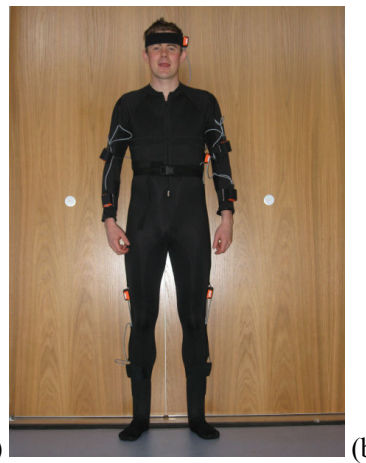

(b)

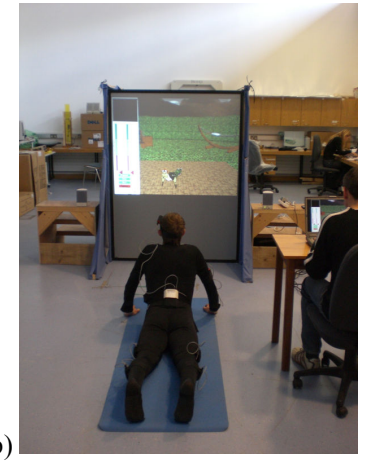

Fig 1. (a) Motion capture suit. (b) Exercise mode

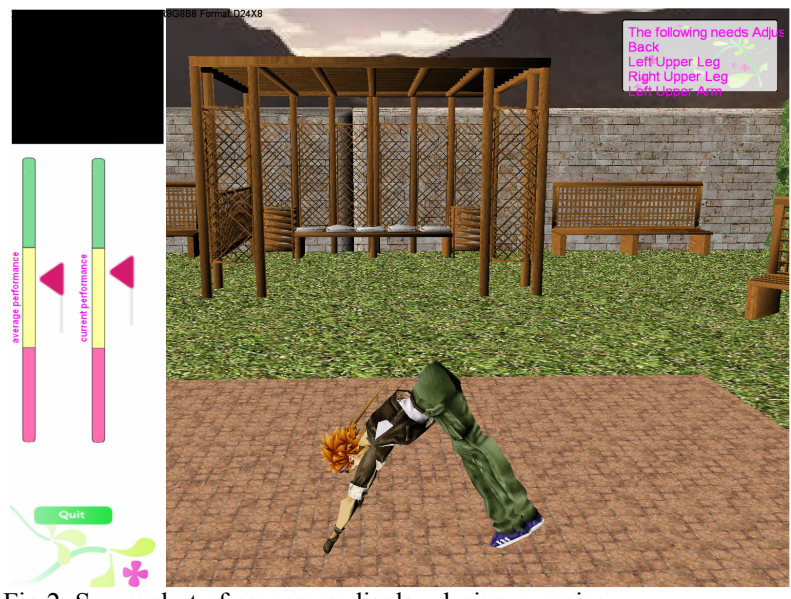

Fig 2. Screenshot of on-screen display during exercise.

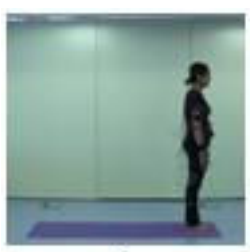

1

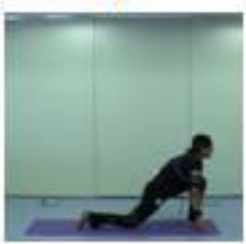

4

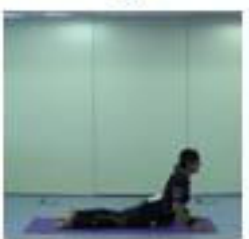

7

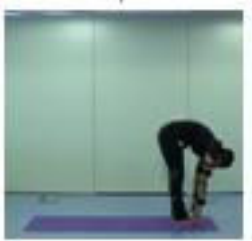

10

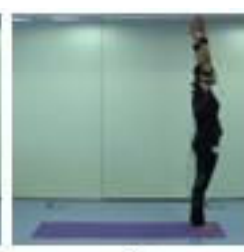

2

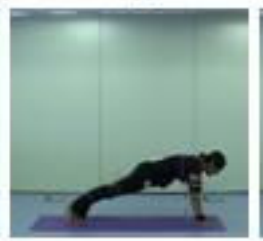

5

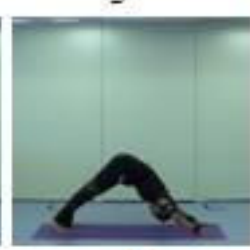

8

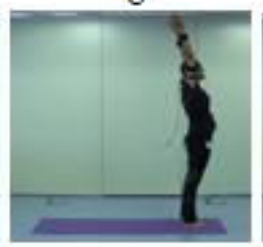

11

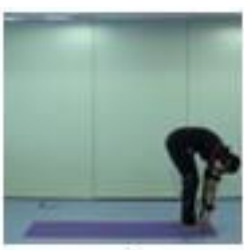

3

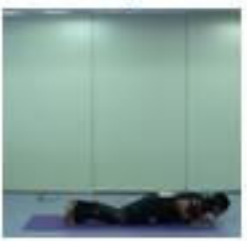

6

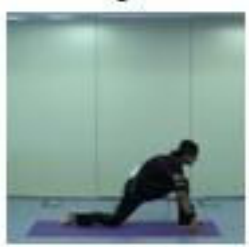

9

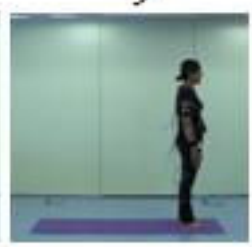

12
Fig 3. The twelve poses of the classical sun salutation sequence.
Using the system begins with a short calibration procedure followed by an audio and visual demonstration of the entire sequence by the VPT. This is effectively a replay of the motion file collected from the expert yoga teacher (perfect movement profile) and users simply view the animated character of the VPT performing the exercise sequence and listens to the audio instructions. When using the system users must copy the on-screen VPT to achieve each of the twelve static poses and audio instruction provides cuing of each pose while music is played in the background. This is achieved by calculating the 1 to 1 euclidean distance measure between the quaternion coordinates of each body segment of the recorded PMP and the live data retrieved from the corresponding MTx motion tracker in the suit. The user is determined to be in the correct pose if each distance is below a set threshold which is set by the therapist prior to commencing the exercise programme. When the users' motion data indicates the correct pose has been achieved the virtual personal trainer will progress onto the next pose. If for any of the poses the user does not satisfactorily achieve the pose within 10 seconds, as compared to the perfect movement profile, audio corrective advice is triggered an projected to the user stating the part of the body needing adjustment e.g. "try bending you left knee a little more". A textbox in the top right corner of the screen also states the body segment requiring adjustment (Fig 2). If after another 10 seconds the correct pose is still not achieved time is up for that pose and the VPT will move on to the next static pose. This process will continue for each individual pose until the sequence is complete. If more than one body segment is incorrect a hierarchical rating decides which body segment receives the feedback as feedback on every segment can be provided. Offline analysis and comparison of performance over successive exercise sessions can also be undertaken.

This paper is broken down into the following sections. The next section will briefly discuss some of the methods for usability evaluation of VRS and Section III will outline the methodology used in our usability evaluation. Section IV will report the results and provide a discussion on the findings and subsequent modifications of the system. Finally Section V will include a conclusion and suggestion for future work.

\section{USABILITY EVALUATION}

Usability is defined as the ability of a system to function effectively, while providing subjective user satisfaction [10]. While a number of methods exist for testing virtual environments [11] they have seldom been reported with VRS. Evaluation can be qualitative, quantitative or a mixture and can involve expert-based or empirical user-based approaches. Types of evaluation methods used include cognitive walkthroughs, formative evaluations, heuristic or guidelines-based expert 
evaluation, post-hoc questionnaire, interview, and finally summative or comparative evaluation [11].

Deutsch and colleagues [12] undertook a formative usability evaluation of the Rutgers Ankle Rehabilitation System by studying therapists who used the system using an ease of use questionnaire and a second questionnaire designed to assess remote monitoring (telerehabilitation) with the system. Weiss and co-workers have used their scenario feedback questionnaire, which is in a modified version of Whitmer and Singer's Presence Questionnaire [13], to assess usability of video-capture virtual rehabilitation systems with neurological patients [14-16].

The usability evaluation of E-Motion was broken into two distinct evaluation stages and certain system modifications were undertaken prior to the second stage. Firstly we conducted an expert walkthrough to identify initial usability problems. Following the first round of system refinements we undertook a user evaluation study with a sample group of healthy novice users.

The expert walkthrough was undertaken with individual "think-aloud" sessions [17]. Expert walkthrough is a formative evaluation method where people who are trained, experienced and knowledgeable in a related area are invited to take the place of would-be users and try to identify possible deficiencies in system design. It is a qualitative observational evaluation and all comments are recorded during testing. Experts voice their thoughts and impressions of the system while they are using the interface and performing the exercises or required tasks. The purpose of an expert walkthrough is to identify initial usability problems, to find defects or omissions in the system, to collect suggestion on how to improve the system, and to consider alterations to the system.

User evaluation was conducted by using VRUSE, a computerised usability questionnaire designed specifically for the evaluation of virtual reality applications [18]. VRUSE is composed of 100 five-point Likert scale-type questions (strongly agree, agree, undecided, disagree, strongly disagree; very satisfactory, satisfactory, neutral, unsatisfactory, very unsatisfactory) divided into 10 separate usability categories:

1. Functionality

2. User Input

3. System Output (Display)

4. User Guidance and Help

5. Consistency

6. Flexibility

7. Simulation Fidelity

8. Error Correction/Handling and Robustness

9. Sense of Immersion/Presence

10. Overall System Usability

Individual sections can be omitted if they are not appropriate to the evaluation context and each section is made up of 6-20 statements. In all questions except the last question in each category responses are rated from strongly agree to strongly disagree. The last question in each category and is rated from very satisfactory to very unsatisfactory. A specific usability score is calculated from the responses to all questions in each category. In addition an overall usability score is calculated from the responses to the last question in each category. An optional freeform comment section is also provided. Kalawsky has previously reported a high reliability level for VRUSE [18].

\section{METHODOLOGY}

\section{A. Expert Walkthrough}

During our expert walkthrough we invited six 'experts' who were highly knowledgeable and were researching or were working in an area related to our VR system design. This group included:

- An ergonomist currently conducting research into human-computer interfaces

- A psychologist currently conducting research into VR for stroke rehabilitation

- An exercise scientist currently conducting research on wearable electrical stimulation devices

- A physiotherapist currently practicing and conducting research in sports and exercise medicine

- A computer scientist currently conducting research in exergaming applications for rehabilitation

- A fulltime yoga teacher and master practitioner

During the expert walkthrough, "think aloud" sessions were conducted with each expert individually in a laboratory setting free from distractions. The experts were instructed to voice any questions, comments or suggestion they had at any point throughout the evaluation. These were noted by the principal researcher during testing and appropriate answers provided.

Evaluation sessions began with the primary researcher presenting an overview of the system and aims of the VR system. Experts were instructed to don the motion capture suit and perform the exercise programme to get a comprehensive idea of all the system features and how a patient would use the setup. Walkthrough session lasted approximately one hour duration. A summary of all usability issues, and suggestions for system improvement were subsequently produced for each expert. When all six experts had completed the walkthrough a list of all recommendation was compiled.

\section{B. User Evaluation Study}

Twelve healthy participants of mean (SD) age of 20.5 (2.24) years of age voluntarily took part in the user evaluation study (six female and six male). Written informed consent was obtained from all subjects. All participants were novice yoga practitioners. An abridged version of the VRUSE usability questionnaire [18] was used for the evaluation. Three subsections (functionality; 
user guidance and help; flexibility) were omitted from testing as these assess the game setup interface which is completed by the therapist in the current VRS setup and in this study by the researcher. This reduced the questionnaire to a total of 80 questions to assess the participants overall experience of using the system.

During testing participants donned the motion capture suit and, following a sensor calibration procedure, completed the exercise sequence twice. All participant questions and comments were noted during evaluation, and appropriate answers provided. Immediately following exercise, the abridged VRUSE questionnaire was completed.

Group mean $( \pm \mathrm{SD})$ specific and overall usability scores were calculated for each category. User comments were tabulated into groups with a common theme/topic.

\section{RESUlTS AND DISCUSSION}

Usability evaluation of E-Motion identified many issues that might cause reduced usability and gathered suggestions to improve our VRS design. The expert walkthrough proved a critical step in assessing usability prior to systematic user evaluation as it identified many issues (Table 1) that could be readily addressed. The VRUSE evaluation provided a benchmark for comparison with similar system and future version of this VRS.

\section{A. Expert walkthrough}

Following completion of the walkthrough we considered all suggestions reported in table 1 but some were not feasible at the current time due to time and cost restrictions but may be investigated at a later date.

To improve the quality of the demonstration exercise sequence (recommendation (REC) 1) we firstly enhanced the accuracy of the MTx inertial motion trackers. A specialized compensation process was applied to each sensor to correct for local magnetic field distortions. The procedure measures the level of magnetic disturbance and yields a new set of electronic data sheet values for each sensor which are stored in the non-volatile memory of the Xsens sensors [19]. Once completed a new expert profile of the sun salutation sequence was re-recorded to provide a higher quality demonstration of the exercise sequence. This procedure also improved the accuracy of the kinematic capture at runtime.

A side on view was provided during poses where the legs move in the saggital plane to address visibility issues with the frontal view in the first version of the system (REC 3). Speakers were implemented, the musical soundtrack was removed, and the audio instructions were re-recorded in order to improve the clarity of the audio feedback during exercise (REC 10 \& 11). A yoga mat was also acquired for the system as advised by the yoga teacher (REC 12).

Some of the recommendation were not carried out at this stage of the project but may be undertaken at a later refinement. On screen text appears in a box in the top right hand corner of the screen as shown in fig 3 . This appears only when a particular body segment requires

TABLE I

SUMMARY OF EXPERTS RECOMMENDATIONS REPORTED DURING EXPERT WALKTHROUGH

\begin{tabular}{|c|c|}
\hline No. & Experts recommendations* \\
\hline & Visual setup \\
\hline 1 & $\begin{array}{l}\text { Re-record the demonstration motion file of the exercise sequence as the current version at times does not show } \\
\text { smooth movement of the avatar between poses. Consider using a different motion capture system if required } \\
\text { such as an optoelectronic system. }\end{array}$ \\
\hline 2 & Reduce the amount of on-screen corrective text during exercise. \\
\hline 3 & Include a side view of on-screen of poses where subjects must move the legs back during the exercise sequence. \\
\hline 4 & Obtain a new on-screen character to improve the graphical appearance of the game \\
\hline 5 & $\begin{array}{l}\text { Use mirror image for the real-time on-screen simulation rather than front on camera view format utilised in the } \\
\text { game. }\end{array}$ \\
\hline 6 & $\begin{array}{l}\text { Use a real-time player's avatar superimposed over the virtual trainer's character during exercise to provide visual } \\
\text { display of current versus required postures. }\end{array}$ \\
\hline 7 & $\begin{array}{l}\text { Use a body segment colour change to provide a visual indication of the part of the body that is incorrect for static } \\
\text { poses during exercise. }\end{array}$ \\
\hline 8 & $\begin{array}{l}\text { Projected the display onto the floor in front of the exercise mat as well as the screen so as subjects can view the } \\
\text { avatar posture during head down yoga poses } \\
\text { Audio setup }\end{array}$ \\
\hline 9 & Include an audio signal or audio instruction when player can move on to the next pose. \\
\hline 10 & Improve sound projection as it is difficult to hear the audio instructions clearly. \\
\hline 11 & Edit the audio instructions to make them more concise, precise and accurate \\
\hline 12 & $\begin{array}{l}\text { User setup } \\
\text { Include a yoga mat for users to stand on during the exercise sequence rather than a conventional exercise mat as } \\
\text { these are too soft and spongy }\end{array}$ \\
\hline
\end{tabular}

*Many comments were repeated by experts so only the common main point is stated once to avoid repetition. 
TABLE II

MEAN AND STANDARD DEVIATION FOR SPECIFIC USABILITY SCORES

\begin{tabular}{llll}
\hline \hline Usability Category & $\mathrm{Max}^{*}$ & $\mathrm{Min}^{+}$ & Score $\pm \mathrm{SD}$ \\
& & & \\
\hline User Input & 70 & 14 & $56.2 \pm 3.6$ \\
System Output & 100 & 20 & $81.9 \pm 6.9$ \\
Consistency & 40 & 8 & $31.3 \pm 3.3$ \\
Simulation Fidelity & 55 & 11 & $42.7 \pm 3.2$ \\
Error Correction & 35 & 7 & $26.8 \pm 2.5$ \\
Sense of Immersion & 50 & 10 & $34.1 \pm 5.9$ \\
Overall System & 55 & 11 & $44.4 \pm 3.9$ \\
Usability & & & \\
\hline \hline
\end{tabular}

*Figures indicates the maximum possible score for individual categories

${ }^{+}$Figures indicates the minimum possible score for individual categories

TABLE III

MEAN AND STANDARD DEVIATION FOR OVERALL USABILITY SCORES

\begin{tabular}{llll}
\hline \hline Usability Category & Max* & Min $^{+}$ & Score \pm SD \\
& & & \\
\hline User Input & 5 & 1 & $4.3 \pm 0.5$ \\
System Output & 5 & 1 & $4.3 \pm 0.5$ \\
Consistency & 5 & 1 & $4.2 \pm 0.7$ \\
Simulation Fidelity & 5 & 1 & $4.0 \pm 0.6$ \\
Error Correction & 5 & 1 & $3.9 \pm 0.7$ \\
Sense of Immersion & 5 & 1 & $3.8 \pm 0.9$ \\
Overall System & 5 & 1 & $4.3 \pm 0.7$ \\
Usability & & & \\
\hline \hline
\end{tabular}

*Figures indicates the maximum possible score for individual categories

${ }^{+}$Figures indicates the minimum possible score for individual categories adjustment as compared to the VPT. If many parts require adjustment there could be up to 5 lines of text on-screen which can take a few seconds to view therefore slowing down the user (REC 2). At this early stage we did not make changes but may restrict text feedback to two lines of text in the future by using a hierarchical ordering as with the audio feedback. Also, we decided against sourcing a new on-screen character at this stage (REC 4). The recommendation to use a mirror image was suggested by a researcher using the Sony EyeToy (Sony Playstation ${ }^{\circledR 2}$ ) for neurological rehabilitation and therefore was biased towards this method of immersion (REC 5). We chose to continue with our current screen projection display setup.

Implementing an avatar of the user superimposed over the virtual personal trainer would possibly have a benefit in this game by providing a useful feedback mechanism to indicate where posture adjustment is required (REC 6). This type of setup was recently reported in a VRS [20] for lower limb rehabilitation where Koritnik described a virtual kinematic model using an optical active marker motion tracking system therefore requiring cameras around the patient A preliminary investigation with healthy subjects demonstrated good adaptation to the virtual environment during simple stepping movements. At this stage it would take too much time but at a later date we would consider exploring this setup.

\section{B. User Evaluation Study}

Mean $( \pm$ SD) VRUSE specific scores are listed in table 2. Overall VRUSE scores are listed in table 3 and user evaluation comments from participants are reported in table 4. The E-Motion system demonstrated a high level of usability in all categories. Overall scores revealed 'sense of immersion' was the lowest and 'error correction' the second lowest overall category. This lack of immersion was also pointed out during the walkthrough when suggestions were made to superimpose a user simulation over the VPT's character as discussed above. Due to the changing dynamic range in specific usability scores comparison between categories is limited

TABLE IV

SUMMARY OF PARTICIPANT COMMENTS AND SUGGESTIONS REPORTED DURING USER EVALUATION

\begin{tabular}{ll}
\hline \hline No. & Participant comment* \\
\hline 1 & Audio feedback was very helpful especially when movements prevented me looking at the screen. \\
2 & The suit was suitable for exercise as it was stretchy therefore the sensors did not restrict my movements. \\
3 & The sensors did not affect my movement. \\
4 & $\begin{array}{l}\text { The text box for correcting positions is difficult to see since it's at the top corner of the screen especially when } \\
\text { you're on the floor and during head down positions. However as long as there is adequate audio input, that }\end{array}$ \\
& $\begin{array}{l}\text { would be more then sufficient } \\
4\end{array}$ \\
5 & I thought that the audio and text feedback was a great idea as compared to an exercise DVD type game. \\
6 & There should be more communication about mistakes between the system and the user. \\
\hline \hline
\end{tabular}

*Many comments were repeated by different evaluators so the common main point is stated once to avoid repetition. 
but can give guidance on usability levels and can certainly be utilised if subsequent versions of E-Motion or similar systems apply the same usability evaluation approach. VRUSE comments provided some positive and negative feedback and highlighted the problem users have viewing the textbox during exercise.

From our experience in undertaking this study we have found that user comments provide essential insight into usability issues. These comments allow evaluators to gather precise user suggestions and problems they encounter during system use. Researchers carrying out these experiments should encourage participants to include comments while completing the VRUSE questionnaire and also probe participants for details of their thoughts on the system following completion of the questionnaire.

\section{CONCLUSION AND FUTURE WORK}

Coupling of the development process and usability testing of virtual rehabilitation systems will assist in maximizing their potential. Through this process improvements can be undertaken on initial prototypes and refinements added as recommended. The VRUSE usability assessment tool can also be used as a benchmark allowing quantitative comparison with subsequent system versions or with other similar systems. Future work with our system is required to carry out an evaluation from a therapist's perspective, integration of a suite of additional therapeutic exercise programmes and testing with a patient population who will use E-Motion as part of their rehabilitation programme.

\section{REFERENCES}

[1] E. A. Keshner, R. V. Kenyon, "Using immersive technology for postural research and rehabilitation" Assistive Technology, Vol. 16, No. 1, pp. 54-62, 2004.

[2] J. E. Deutsch, J. Latonio, G. C . Burdea, R. Boian, "Post stroke rehabilitation with Rutgers Ankle System: a case study", Presence: Teleoperators and Virtual Environments, Vol. 10, No. 4, pp. 416430, 2001.

[3] M. K. Holden, A. Dettwiler, T. Dyar, G. Niemann, E. Bizzi, "Retraining movement in patients with acquired brain injury using a virtual environment", Studies in Health Technology and Informatics, pp192-8, 2001.

[4] S. V. Adamovich, A. S. Merians, R. Boian, M. Tremaine, G. S. Burdea, M. Recce, H. Poizner, "A virtual reality based exercise system for hand rehabilitation post stroke", In proceedings of Second International Workshop on Virtual Rehabilitation: Piscataway, pp. 74-81, September 21-22, 2003.

[5] M. K. Holden, T. Dyar, "Virtual environment training: a new tool for rehabilitation", Neurology Report, Vol. 26, pp. 62-71, 2002.

[6] H. Sveistrup, "Motor rehabilitation using virtual reality", $J$ Neuroengineering and Rehabilitation, Available: http://www.jneuroengrehab.com/content/1/1/10

[7] J. L. Jordan, N. E. Foster, M. A. Holden, E. E. J. Mason, "Interventions to improve adherence to exercise for chronic musculoskeletal pain in adults (protocol)" Cochrane Database of Systematic Reviews, Issue 2. Art. No.: CD005956. DOI: 10.1002/14651858.CD005956, 2006.

[8] D Kelly, D Fitzgerald, J Foody, D Kumar, T Ward, B Caulfield, C Markham. "The E-Motion System: Motion Capture and Movement-based Biofeedback Game" in Proc. 9th International Conference on computer Games, Dublin, pp. 19-23, 2006.
[9] J. A. Raub, "Psychophysiological effects of Hatha yoga on musculoskeletal and cardiopulmonary Function: a literature review", The Journal of Alternative and Complementary Medicine, Vol. 8, No. 6, pp. 767-812, 2002.

[10] ISO 9241 - International Standardization Organization, "Ergonomic requirements for office work and visual display terminals (VDT's)", 1998.

[11] D. A. Bowman, J. L. Gabbard, D. Hix, "A Survey of Usability in Virtual Environments: Classification and comparison of methods," Presence, Vol. 11, No. 4, pp. 404-424, 2002.

[12] J. A. Deutsch, J. A. Lewis, E. Whitworth, R. Boian, G. Burdea, M. Tremaine, "Formative evaluation and preliminary findings of a virtual reality telerehabilitation system for lower extremity", Presence, Vol. 14, No. 2, pp. 198-213, 2005.

[13] B. G. Witmer, M. J. Singer, "Measuring presence in virtual environments: a presence questionnaire", Presence: Teleoperators and Virtual Environments, Vol. 7, No. 3, pp. 225-240, 1998.

[14] R. Kizony, N. Katz, P. L. Weiss, "Adapting an immersive virtual reality system for rehabilitation", The Journal of Visualization and Computer Animation, Vol. 14, pp. 261-268, 2003.

[15] H. Brown-Rubin, R. Kizony, P. L. Weiss, "Effect of movement constraint within virtual environments", The Israel Journal of Occupational Therapy, Vol. 14, No. 2, 2005.

[16] U. Feintuch, et al, "Integrating Haptic-tactile Feedback into a Video-Capture-Based Virtual Environment for Rehabilitation", CyberPsychology \& Behaviour, Vol. 9, No. 9, pp. 129-132, 2006.

[17] D. Hix, H. R. Hartson, "Developing user interfaces: Ensuring usability through product and process", John Wiley \& Sons 1993.

[18] R. S. Kalawsky, "VRUSE-a computerised diagnostic tool: for usability evaluation of virtual/synthetic environment systems", Applied Ergonomics, Vol. 30, pp. 11-25, 1999.

[19] Xsens Technologies, "Magnetic Field Mapper MT Software Add User Manual", Documant MT0202P, Revision B, January 29, 2003.

[20] T. Koritnik, T. Bajd, M. Munih, "Virtual environment for lowerextremities training" Gait \& Posture, Vol. 27, No. 2, 323-330, 2008. 